\title{
Noises and Signal-to-Noise Ratio of Nanosize EIS and ISFET Biosensors
}

\author{
Lusine Gasparyan"1,2, Ilya Mazo3 ${ }^{3}$, Vahan Simonyan1, Ferdinand Gasparyan ${ }^{2 *}$ \\ ${ }^{1}$ DNA-HIVE LLC 15313 Diamond Cove Terrace, Rockville, USA \\ ${ }^{2}$ Yerevan State University, Yerevan, Armenia \\ ${ }^{3}$ Argentis LLC, Gaithersburg, USA \\ Email: ${ }^{\star}$ fgaspar@ysu.am
}

How to cite this paper: Gasparyan, L., Mazo, I., Simonyan, V. and Gasparyan, F. (2020) Noises and Signal-to-Noise Ratio of Nanosize EIS and ISFET Biosensors. Open Journal of Biophysics, 10, 1-12. https://doi.org/10.4236/ojbiphy.2020.10100 $\underline{1}$

Received: November 30, 2019

Accepted: December 17, 2019

Published: December 20, 2019

Copyright (c) 2020 by author(s) and Scientific Research Publishing Inc. This work is licensed under the Creative Commons Attribution International License (CC BY 4.0).

http://creativecommons.org/licenses/by/4.0/

\begin{abstract}
The results of comparative theoretical analyzes of the behavior of internal low-frequency noises, signal-to-noise ratio and sensitivity to DNA molecules for EIS and ISFET based nanosize biosensors are presented. It is shown that EIS biosensor is more sensitive to the presence of DNA molecules in aqueous solution than ISFET sensor. Internal electrical noises level decreases with the increase of concentration of DNA molecules in aqueous solution. In the frequency range $10^{-3}-10^{3} \mathrm{~Hz}$ noises level for EIS sensor about in three orders is higher than for ISFET sensor. In the other hand, signal-to-noise ratio for capacitive EIS biosensor is much higher than for ISFET sensor.
\end{abstract}

\section{Keywords}

Biosensor, Noise, Sensitivity, Signal-to-Noise Ratio

\section{Introduction}

Biosensors based on the field-effect transistors (BioFETs) are potential candidates for future bioassay applications due to its fast response, high sensitivity, high signal-to-noise ratio, small sensing size and low cost. The ion-sensitive field-effect transistors (ISFET) and electrolyte-insulator-semiconductor (EIS) $\mathrm{pH}$-sensitive structures are very important sensors for in vivo continuous monitoring application of physiological and environmental mediums and systems. Main types of semiconductor based BioFET sensors operated using peculiarities of field-effect, especially modulation of depletion layer conductance on the semiconductor-other media (metal, dielectric) interface. It is obvious, that sensitivity, selectivity and detectivity of electronic devices, including BioFET sensors, determined in general by the internal electrical noises types, its level and fre- 
quency behavior, and consequently by the signal-to-noise ratio (SNR).

Fluctuations in environmental parameters, such as, for example, the concentration and velocity of ions and DNA molecules in an aqueous solution, lead to important random scattering processes that can affect the viability of sequencing. A simple model that captures the role of complex environment in electronic de-phasing and its ability to remove charge carriers from current-carrying states is analyzed in [1]. The environment is composed of ionic, DNA and aqueous solution fluctuations and other random excitations that may drastically affect the electron dynamics, and thus the ionic (or tunnel) current and noise at the DNA detection and sequencing processes [2].

Note that the excess noise level at the DNA sequencing using the solid-state nanopores (which a few tens of pA to $100 \mathrm{pA}, 10$ times larger than that of protein counterparts [3] [4] [5] [6]) has been one of the key issues responsible for the degraded SNR and temporal and spatial resolution.

Previous theoretical works showed the four DNA nucleotides possess statistically distinguishable electronic signatures in the form of ionic blockade or tunnel current distributions when accounting for structural distortions and partial control of the DNA dynamics [7] [8] [9] [10] [11]. These results indicate DNA sequencing is, in principle, possible via transverse current measurements. However, such studies have neglected scattering processes, such as fluctuations of the environment, which introduce current noise, and may thus affect the ability to distinguish the DNA bases. A solid-state nanopore platform with a low noise level and sufficient sensitivity to discriminate single-strand DNA (ssDNA) homopolymers of poly- $\mathrm{A}_{40}$ and poly- $\mathrm{T}_{40}$ using ionic current blockade sensing is proposed and demonstrated in [12].

In Ref. [13] the low-frequency pH-dependent electrochemical noise that originates from the ionic conductance of the electrode-electrolyte-FET structure of the device and that the noise depends on the concentration of the electrolyte and $1 / f$ in nature are investigated. The statistical and frequency analysis of this electrochemical noise of a commercial ISFET sensor, under room temperature has been performed for different $\mathrm{pH}$ values. It is also proposed a concentration dependent $a / f$ and $b / f^{2}$ model of the noise with different values of the coefficients $a$ and $b$ (here $f$ is the frequency).

The numerical and analytical theory of signal and noise of double-gated $\mathrm{pH}$-sensors was provided in [14]. The transport and noise properties of an array of silicon nanowire FET sensors are investigated in [15]. It is shown that drain current substantially depends on $\mathrm{pH}$ value and SNR reaches the high value of $10^{5}$. The noise characteristic index decreases from 1.1 to 0.7 with the growth of the liquid gate voltage. Noise behavior is successfully explained in the framework of the correlated number-mobility unified fluctuation model.

To explain the nature and behavior of $1 / f$-noise in devices based on FET, the following basic theories and models are proposed: the carrier density or number fluctuation model introduced by McWhorter [16], the carriers mobil- 
ity fluctuation model proposed by Hooge empirical relation [17], the electron-phonon interaction model [18] [19] [20] [21] and the charge fluctuation model [22] [23] proposed by us.

The accuracy of ISFET output measurement is greatly affected by the presences of internal noises, drift, diffusion and slow response of the device. Although the noise analysis of ISFETs so far performed in different literature relates only to sources originated from FET structure which is almost constant for a particular device, the $\mathrm{pH}$, or charged DNA molecules concentration dependent electrochemical noise has not been substantially explored and analyzed in detail.

As usually the time constants involved in the detection of biological and chemical species in electrolyte medium via field effect are relatively large, it would be expected that low-frequency noise is more critical than other types of noises in BioFET sensors. Therefore, further we will analyze just low-frequency $1 / f$-noise behavior.

In this paper the results of theoretical simulation of the behavior and peculiarities of low-frequency $1 / f$-noise and signal-to-noise ratio for nanosize BioFET sensors are presented. Numerical simulation of the noise spectral density and signal-to-noise ratio we do for EIS and ISFET sensors based on the silicon (as semiconductor) and silicon dioxide (as insulator).

\section{Low-Frequency Noises in BioFET Sensors}

The detailed analysis shows that main types of electrical noises in BioFET sensors can be classified as follows [24]-[29]:

1) Noise generated in solid state part of the sensor

- Thermal noise;

- Generation-recombination (g-r) noise in the space charge region at the substrate-channel interface;

- $1 / f$-and g-r noises generated due to trapping and detrapping on the semiconductor-insulator interface;

- Hooge's bulk $1 / f$-noise in semiconductor;

- Current channel $1 / f$-noise.

2) Electrochemical noise associated with the ion/charged molecules-insulator interactions

- Thermal noise;

- $1 / f$-noise in corrosive interfaces;

- Shot or Schottky noise;

- Spurious noise.

3) Noise generated in the aqueous solution and at the reference electrode as well as noise resulting from the fluctuations of the biasing elements

- Bulk thermal noise;

- Diffusion layer thermal noise;

- $1 / f$-noise in corrosive interfaces;

- Biological noise. 
Low-frequency noise conditioned by the random fluctuations of concentration or mobility of current carriers, ions and charged DNA molecules in aqueous solution, by the trapping-detrapping processes on the surface and interface states, by the electron-phonon interactions, as well as by the fluctuation of electron's and phonon's distribution functions in the bulk of semiconductor. As already noted, for BioFET sensors low-frequency noise is of greater interest, which, as a rule, can be determined and explained using the Hooge's model [21] [30] [31] [32], McWhorter or correlated number-mobility fluctuation model [16] [33] [34] and charge fluctuation model [22].

For the linear regime of operation of BioFETs signal-to-noise ratio can be calculated using expressions:

$$
S N R=\frac{I_{S}}{I_{N}}=\frac{I_{S}}{\sqrt{S_{I} \Delta f}}
$$

or

$$
=\frac{V_{S}}{V_{N}}=\frac{V_{S}}{\sqrt{S_{V} \Delta f}} .
$$

Here $I_{S}\left(V_{S}\right)$ and $I_{N}\left(V_{N}\right)$ are useful signal current (voltage) and noise equivalent current (voltage), correspondingly, $S_{I}\left(S_{V}\right)$ are current (voltage) noise spectral densities, $\Delta f$ is the frequency bandwidth. In numerical calculations we take $\Delta f=1 \mathrm{~Hz}$.

\subsection{EIS Biosensor}

It is clear that for EIS biosensors main physical effects that influence on the low-frequency noise behavior is carried out in the interface electrolyte-insulator (see Figure $8 \mathrm{~b}$ in [35]). As EIS is the capacitive device the main type of electrical noise must be connected with capacitance (or charge) random fluctuation. At such situation dominant noise type will be noise conditioned by the oxide (insulator) surface charge fluctuation. We will take accounts that the oxide surface charge is changed during the DNA sensing processes. This is occurring by the capture of negatively charged DNA molecules on the proton acceptor $\mathrm{OH}_{2}^{+}$ bonds and the capture of $\mathrm{H}^{+}$ions (protons) of the solution on the proton donor free $\mathrm{OH}^{-}$bonds on the interface solution-insulator (see Figure 2 in [7], and [36]). These are molecules and ions located at a distance of Debye length from the oxide surface. Thus, to calculate noise spectral density, we can use an expression that takes into account charge fluctuations [37]:

$$
S_{V}(f)=\frac{S_{Q}(f)}{C_{e f}^{2}}=\frac{e^{2} N_{t}}{w l C_{e f}^{2}} \frac{1}{f} .
$$

Here $S_{Q}(f)$ is the noise spectral density conditioned by charge fluctuation, $N_{t}$ is the equivalent total density of traps per unit area at the $\mathrm{SiO}_{2} /$ electrolyte interface, $w$ and $l$ are width and length of the sensitive oxide layer, correspondingly, $f$ is the frequency, $C_{e f}=\frac{C_{o x} C_{d}}{C_{o x}+C_{d}}, C_{o x}$ and $C_{d}$ are the capaci- 
tances per unit area of the insulator layer and the semiconductor depletion layer, correspondingly:

$$
C_{o x}=\frac{\varepsilon_{0} \varepsilon_{o x}}{t_{o x}}, C_{d}=\frac{\varepsilon_{0} \varepsilon_{S i}}{t_{d}} .
$$

Here $\varepsilon_{0}, \varepsilon_{o x}$ and $\varepsilon_{S i}$ are dielectric permittivity of free space, insulator and semiconductor, $t_{o x}$ and $t_{d}$ are thicknesses of insulator and semiconductor depletion layers, correspondingly. For simplicity assume that before fill the aqueous solution by the DNA molecules, the proton donor $\mathrm{OH}^{-}$bonds in $\mathrm{SiO}_{2} /$ electrolyte interface was fully compensated (passivized) by the protons of the solution and in further do not participate on the surface charge changing process. Therefore the oxide surface charge per unit area conditioned only by proton acceptor $\mathrm{OH}_{2}^{+}$bonds $\left(Q_{o x}=q N_{t}^{+}\right)$will be changed only at the capture of negatively charged DNA molecules. So it becomes (see [7] [36]):

$$
Q_{o X}^{\prime}=q N_{t}^{+}(1-\delta), \quad \delta \equiv \frac{N_{D N A}}{N_{t}^{+}} .
$$

Here $N_{t}^{+}$is the concentration of the proton acceptor traps on the unit area of oxide surface, $N_{D N A}$ is the DNA surface concentration in solution near the oxide at a distance of the Debye length (see also [7]). Thus Equation (2) will be changed as follows:

$$
S_{V}(f)=\frac{e^{2} N_{t}^{+}(1-\delta)}{w l C_{e f}^{2}} \frac{1}{f} .
$$

For numerical simulation we used following parameters: $t_{d}=2 \times 10^{-6} \mathrm{~cm}$, $t_{o x}=10^{-6} \mathrm{~cm}, \varepsilon_{S i}=11.7, \varepsilon_{o x}=3.9, \quad \varepsilon_{0}=8.85 \times 10^{-14} \mathrm{~F} / \mathrm{cm}, \quad w=1.5 \times 10^{-6} \mathrm{~cm}$; $l=2 \times 10^{-6} \mathrm{~cm}$. For the value of $N_{t}^{+}$we can do following estimation. Assume that traps concentration on the interface $\mathrm{SiO}_{2}$-electrolyte same than $\mathrm{SiO}_{2}$ - $\mathrm{Si}$ interface. According data [7] [37] traps concentration in $\mathrm{Si}-\mathrm{SiO}_{2}$ interface is about $\left(10^{10}-10^{11}\right) \mathrm{cm}^{-2}$. In the numerical calculations we will use $N_{t}^{+} \approx 10^{11} \mathrm{~cm}^{-2}$. For $C_{e f}$ we have $C_{e f} \approx 2.1 \times 10^{-5} \mathrm{~F} / \mathrm{cm}^{2}$.

As we can see from expression (4) increase of the sensitive surface area ( $w l)$ brings to decrease of the noise level. On the other hand it is well known that increase of sensitive surface brings to increase of sensitivity of the BioFET to DNA molecules (see also [7]). At the same time, for low noise and high sensitivity, sensors with a relatively large surface area should be used.

In Figure 1 spectral dependency of low-frequency noise for EIS biosensor is presented for different values of DNA concentration (or $\delta$ ) in logarithmic scale. Noises level decrease with increasing of the DNA concentration. Such behavior can be explained as follows. Increasing of the DNA molecules number near oxide surface brings to partially compensation of surface positive charge. As a result charge fluctuation level and consequently noise spectral density of this charge is decrease.

As it is follow from Equation (4) at the completely compensation (passivation) of oxide surface charge ( $\delta=1$ ) noise density of charge fluctuation becomes to 
zero. Now total noise will be determined by the other types of noises (thermal, $1 / f$-noise in corrosive interfaces, spurious noise). Dependencies of noise level from DNA molecules concentration (or $\delta$ ) presented in Figure 2. Noise spectral density decreases when DNA concentration is increase. As it is shown in

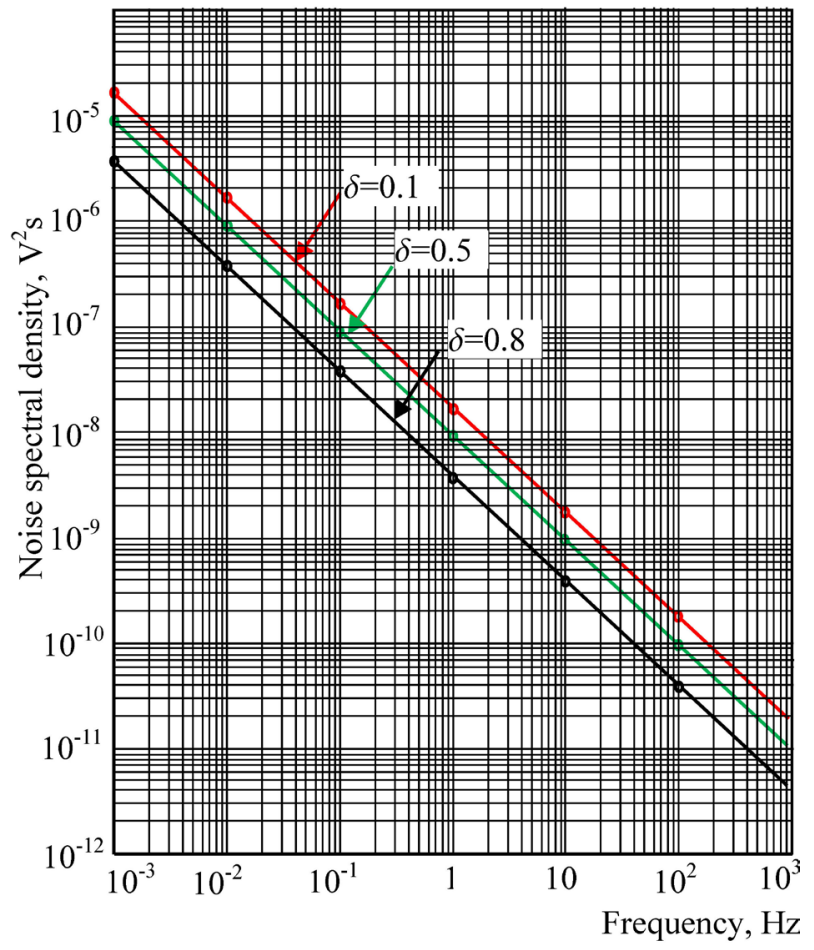

Figure 1. Low-frequency noise spectral dependency for EIS biosensor. The graphs are built according Equation (4).

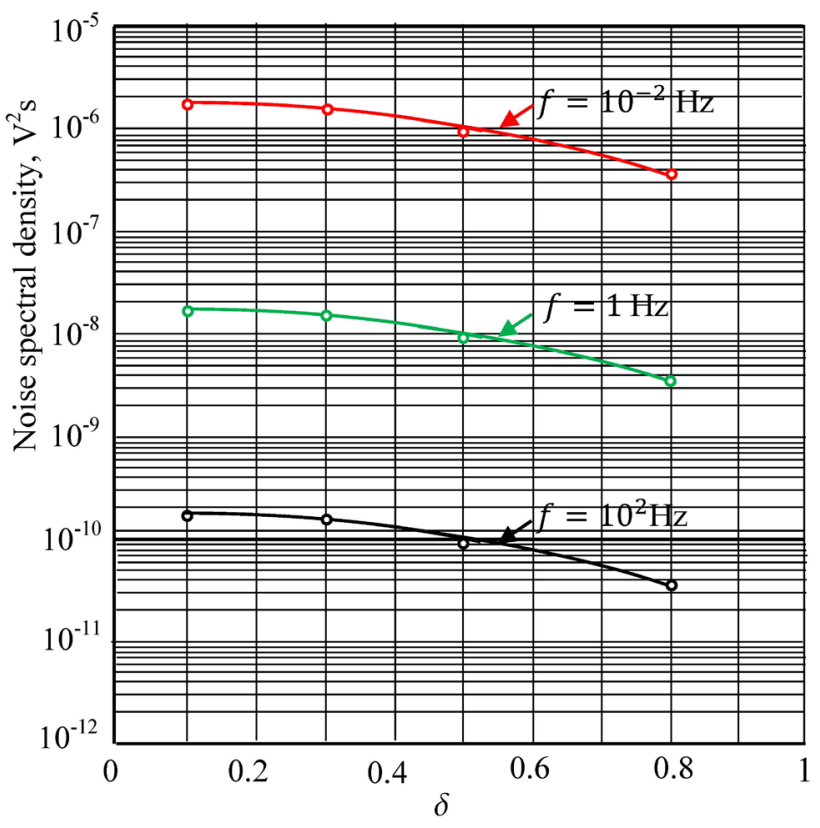

Figure 2. Dependency of noise spectral density for EIS biosensor vs. concentration of DNA molecules. 
Figure 3 for the parameters (sizes) of the EIS biosensor selected above SNR can reach high values up to $10^{5}-10^{6}$. Note that SNR is calculated according to formula (1b).

\subsection{ISFET Biosensor}

The main physical processes that occur in ISFET sensors and affect the response of the device to the presence of DNA molecules in an aqueous solution and to the source of generated noise in the channel occur mainly in the current channel between sources and drain electrodes (see Figure 1a in [7]). Fluctuation of the source-drain current besides carrier's concentration and mobility fluctuation is conditioned also by the fluctuation (change) of the semiconductor surface potential that depends on concentration of DNA molecules in aqueous solution via charge fluctuation in $\mathrm{SiO}_{2}$ /electrolyte interface [7] [36]. According to Hooge's empirical mobility fluctuation model low-frequency noise for ISFET sensor can be presented by the following expression:

$$
S_{i}(f)=\frac{e \alpha_{H} I_{s d}}{w l C_{o x}\left(V_{g}-V_{t h}\right)} \frac{1}{f} .
$$

Here $V_{g}$ and $V_{t h}$ are gate ${ }^{1}$ and threshold voltages, $I_{s d}$ is the source-drain current of field-effect transistor, $\alpha_{H}$ is the Hooge's parameter. For ISFET biosensor in [7] we obtain an expression for the source-drain current, which, in addition to the electrical and structural parameters of the semiconductor, also depends on the surface potential of current channel (semiconductor depletion layer) (see Equation (3) in [7]). After some modifications, source-drain current can be represented by the following form:

$$
\begin{aligned}
I_{s d} \approx & \frac{e t_{d} w n_{0} V_{d s}}{l \varphi_{T}}\left[\mu_{0}-\theta\left(V_{g}+2 \varphi_{F}+\phi_{d l}-\frac{\Phi_{S i}-\Phi_{o x}}{q}+\frac{Q_{o x}}{C_{o x}}\right)\right] . \\
& \times\left[\varphi_{T}\left(1+\ln \frac{B}{2}\right)+V_{g}+2 \varphi_{F}+\phi_{d l}-\frac{\Phi_{S i}-\Phi_{o x}}{q}+\frac{Q_{o x}}{C_{o x}}\right]
\end{aligned}
$$

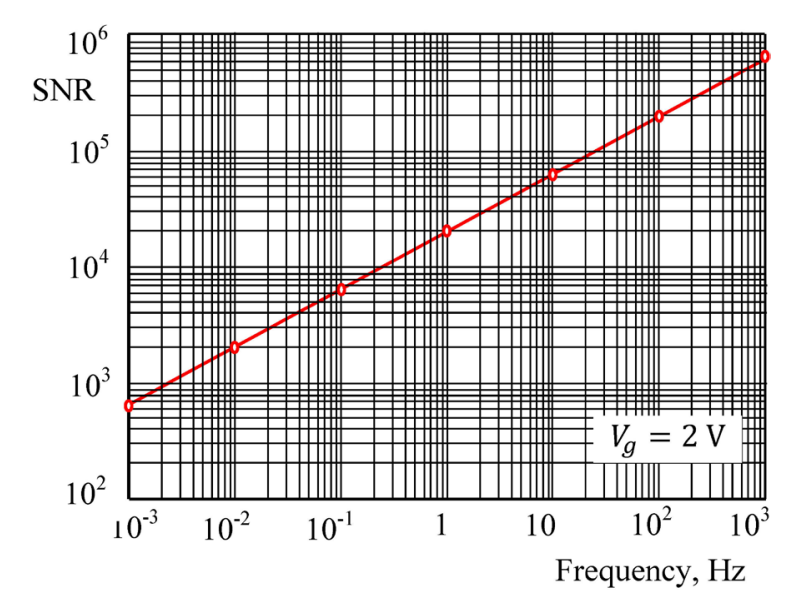

Figure 3. Frequency dependency of SNR for EIS biosensor at the gate voltage $2 \mathrm{~V}$.

${ }^{1}$ Under gate voltage we mean voltage applied between reference electrode and source electrode. 
Here

$$
\begin{gathered}
\varphi_{F}=2 \varphi_{T} \ln \frac{N_{A}}{n_{i}}, \varphi_{T}=\frac{k_{B} T}{q}, \phi_{d l}=2 \varphi_{T}\left(\frac{\varepsilon_{w}}{\varepsilon_{r}} \frac{N_{s o l}}{K_{A K}^{+}+H_{s}^{+}}\right) ; \phi_{o x}=\frac{q N_{t}}{C_{o x}}, \\
C_{o x}=\frac{\varepsilon_{0} \varepsilon_{o x}}{t_{o x}} ; B \equiv \frac{\varphi_{T} \varepsilon_{0} \varepsilon_{o x} N_{A}}{q t_{o x}^{2} n_{i}^{2}} .
\end{gathered}
$$

Here $\phi_{o x}$ and $\phi_{d l}$ are potentials of the oxide layer and double layer, correspondingly, $\Phi_{S i}$ and $\Phi_{o x}$ are the work functions of silicon and silicon dioxide $\left(\mathrm{SiO}_{2}\right)$, correspondingly; $V_{d s}$ is the source-drain voltage, $Q_{o x}$ is the oxide layer charge per unit area, $C_{o x}$ is the capacitance of the oxide layer per unit area; $\varepsilon_{w}$ and $\varepsilon_{r}$ are the dielectric permittivity of water and electrolyte, respectively; $N_{A}$ is the doping acceptor concentration in p-Si substrate; $n_{i}$ is the intrinsic carrier concentration in bulk silicon, $n_{0}$ and $p_{0}\left(p_{0} \approx N_{A}\right)$ are the equilibrium electron and hole concentrations in semiconductor, $K_{A K}^{+}$is the molar concentration of the cations in the solution, $\mathrm{H}_{s}^{+}$is the molar concentration of the hydrogen ions (protons) at the oxide surface, $k_{B}$ is the Boltzmann constant, $T$ is the absolute temperature, $\mu_{0}$ is the low-field magnitude of the mobility of carriers, $\theta$ is the some constant.

For numerical simulation besides of parameters presented above for EIS sensor we use also following additional parameters: $\mu_{0}=260 \mathrm{~cm}^{2} /(\mathrm{V} \cdot \mathrm{s})$ [38], $\theta=28 \mathrm{~cm}^{2} /\left(\mathrm{V}^{2} \cdot \mathrm{s}\right) \quad$ [39] [40] [41], $\varphi_{T}=0.026 \mathrm{~V}, N_{\text {sol }}=0.015 \mathrm{~mol} / \mathrm{l}$ [41], $K_{A K}^{+}=0.001 \mathrm{~mol} / \mathrm{l} \quad[41], \varepsilon_{w} \approx 80, \varepsilon_{r} \approx 78, \Phi_{S i}=4.85 \mathrm{eV} \quad[37], \Phi_{o x}=5 \mathrm{eV}$ [37], $\quad N_{A}=10^{15} \mathrm{~cm}^{-3}, \quad N_{V}=2.5 \times 10^{19} \mathrm{~cm}^{-3} \quad\left(m_{n}^{*}=m_{p}^{*} \equiv m_{0}=9.1 \times 10^{-31} \mathrm{~kg}, m_{n}^{*}\right.$ and $m_{p}^{*}$ are effective mass of electrons and holes, $m_{0}$ is the free electron mass).

In Figure 4 spectral dependency of low-frequency noise for ISFET biosensor

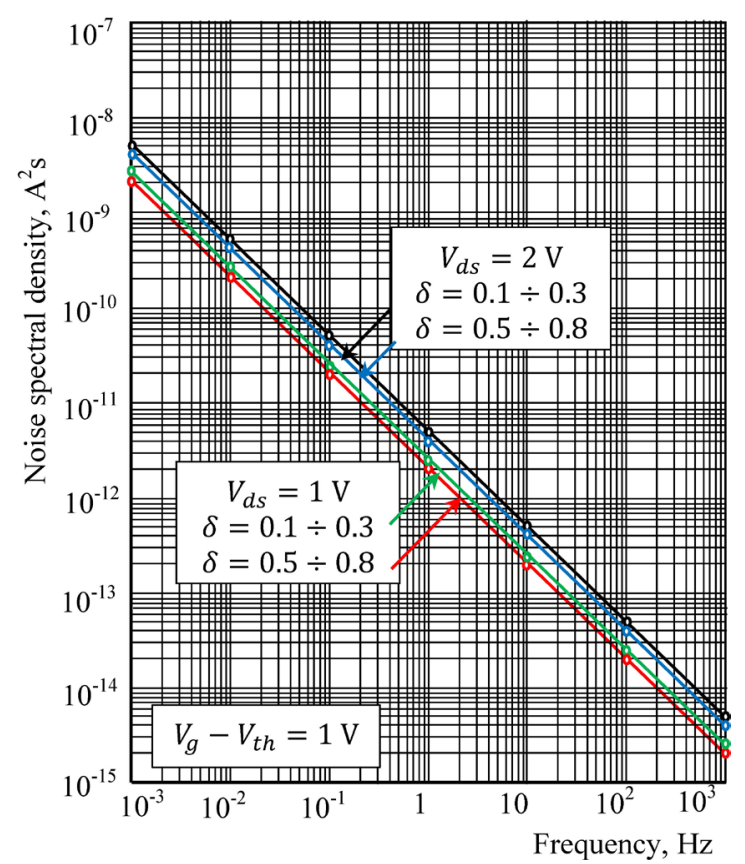

Figure 4. Spectral dependency of noise spectral density for ISFET biosensor according Equation (7). 


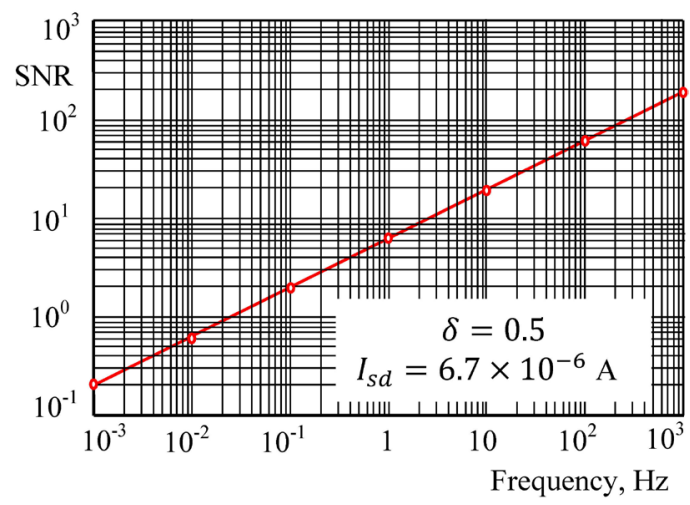

Figure 5. Frequency dependency of signal-to-noise ratio for ISFET biosensor.

are presented in logarithmic scale. Increasing both source-drain voltage and concentration of DNA molecules brings to weak grow of the noise level. Magnitude of the signal-to-noise ratio for ISFET sensor in several orders is smaller than for EIS sensor (see Figure 3 and Figure 5).

\section{Conclusion}

EIS biosensor is more sensitive to DNA molecules than ISFET sensor. It is conditioned by the more strong modulation of the oxide charge (EIS capacitance) in comparison with weak modulation of the source-drain current of ISFET under influence of DNA molecules. Low-frequency noises level decreases with increasing of concentration of DNA molecules in solution. Such behavior conditioned by the growing dependency of source-drain current with increase of concentration of DNA molecules ${ }^{2}$. High sensitivity of the EIS biosensor conditioned by the "deep modulation" of the charge of oxide layer and consequently by the deep modulation of the EIS totals capacitance under influence of charged DNA molecules in aqueous solution.

\section{Author Contribution Information}

All authors participated in the statement of the problem and discussion of the results. L. Gasparyan, and F. Gasparyan conducted a literature review. F. Gasparyan and V. Simonyan made calculations and participated in the writing of the text of the article.

\section{Conflicts of Interest}

The authors declare no conflicts of interest regarding the publication of this paper.

\section{References}

[1] Krems, M., Zwolak, M., Pershin, Y.V. and Di Ventra, M. (2009) Effect of Noise on DNA Sequencing via Transverse Electronic Transport. Biophysical Journal, 97, 1990-1996. https://doi.org/10.1016/j.bpj.2009.06.055

${ }^{2}$ Explanation of such behavior gives in Ref. [2]. 
[2] Di Ventra, M. (2008) Electrical Transport in Nanoscale Systems. Cambridge University Press, Cambridge. https://doi.org/10.1017/CBO9780511755606

[3] Wanunu, M., Dadosh, T., Ray, V., Jin, J., McReynolds, L. and Dmdich, M. (2010) Rapid Electronic Detection of Probe-Specific microRNAs Using Thin Nanopore Sensors. Nature Nanotechnology, 5, 807-814. https://doi.org/10.1038/nnano.2010.202

[4] Garaj, S., Hubbard, W., Reina, A., Kong, J., Branton, D. and Golovchenko, J.A. (2010) Graphene as a Subnanometre Trans-Electrode Membrane. Nature, 467, 190-193. https://doi.org/10.1038/nature09379

[5] Merchant, C.A., Healy, K., Wanunu, M., et al. (2010) DNA Translocation through Graphene Nanopores. Nano Letters, 10, 2915-2921. https://doi.org/10.1021/nl101046t

[6] Schneider, G.F., Kowalczyk, S.W., Calado, V.E., Pandraut, G., et al. (2010) DNA Translocation through Graphene Nanopores. Nano Letters, 10, 3163-3167. https://doi.org/10.1021/nl102069z

[7] Gasparyan, L., Mazo, I., Simonyan, V. and Gasparyan, F. (2019) ISFET Based DNA Sensor: Current-Voltage Characteristic and Sensitivity. Open Journal of Biophysics, 9, 239-253. https://doi.org/10.4236/ojbiphy.2019.94017

[8] Zwolak, M. and Di Ventra, M. (2005) Electronic Signature of DNA Nucleotides via Transverse Transport. Nano Letters, 5, 421-424. https://doi.org/10.1021/nl048289w

[9] Lagerqvist, J., Zwolak, M. and Di Ventra, M. (2006) Fast DNA Sequencing via Transverse Electronic Transport. Nano Letters, 6, 779-782. https://doi.org/10.1021/nl0601076

[10] Lagerqvist, J., Zwolak, M. and Di Ventra, M. (2007) Influence of the Environment and Probes on Rapid DNA Sequencing via Transverse Electronic Transport. Biophysical Journal, 93, 2384-2390. https://doi.org/10.1529/biophysj.106.102269

[11] Lagerqvist, J., Zwolak, M. and Di Ventra, M. (2007) Comment on Characterization of the Tunneling Conductance across DNA Bases. Physical Review E: Statistical, Nonlinear, and Soft Matter Physics, 76, Article ID: 013901. https://doi.org/10.1103/PhysRevE.76.013901

[12] Lee, M.-H., Kumar, A., Park, K.-B., Cho, S.-Y., Kim, H.-M., Lim, M.-C., Kim, Y.-R. and Kim, K.-B. (2014) A Low-Noise Solid-State Nanopore Platform Based on a Highly Insulating Substrate. Scientific Reports, 4, Article No. 7448. https://doi.org/10.1038/srep07448

[13] Das, M.P. and Bhuyan, M. (2013) Modeling of pH Dependent Electrochemical Noise in Ion Sensitive Field Effect Transistors ISFET. Sensors and Transducers, 149, 102-108. https://doi.org/10.4236/mnsms.2014.43013

[14] Tsividis, Y. (1999) Operation and Modeling of the MOS Transistor. 2nd Edition, McGraw-Hill, New York.

[15] Gasparyan, F., Khondkaryan, H., Arakelyan, A., Zadorozhnyi, I., Pud, S. and Vitusevich, S. (2016) Double-Gated Si NW Sensors: Low-Frequency Noise and Photoelectric Properties. Journal of Applied Physics, 120, Article ID: 064902(1-8). https://doi.org/10.1063/1.4960704

[16] McWhorter, A.L. (1957) 1/f Noise and Germanium Surface Properties. In: Kingston, R.H., Ed., Semiconductor Surface Physics, University of Pennsylvania Press, Philadelphia, 207-228.

[17] Hooge, F.N. (1993) 1/f Noise Sources. IEEE Transactions on Electron Devices, 41, 1926-1935. https://doi.org/10.1109/16.333808 
[18] Gasparyan, F.V., Melkonyan, S.V. and Asriyan, H.V. (2005) Space Confined and Bulk Temporal Fluctuations of Phonons and Linkage between Two Models of $1 / \mathrm{f}$ Noise in Semiconductors. Semiconductor Micro- and Nano-Electronics, Aghveran, 16-18 September 2005, 24-27.

[19] Melkonyan, S.V., Gasparyan, F.V., Aroutiounian, V.M. and Asriyan, H.V. (2005) 1/f Type Noise in View of Phonons Interface Percolation Dynamics. Noise and Fluctuations, Salamanca, 19-23 September 2005, Vol. 780, 87-91.

https://doi.org/10.1063/1.2036705

[20] Melkonyan, S.V., Aroutiounian, V.M., Gasparyan, F.V. and Asriyan, H.V. (2006) Phonon Mechanism of Mobility Equilibrium Fluctuation and Properties of 1/f-Noise. Physica, B: Physics of Condensed Matter, 382, 65-70. https://doi.org/10.1016/j.physb.2006.01.521

[21] Melkonyan, S.V., Gasparyan, F.V. and Asriyan, H.V. (2007) Main Sources of Electron Mobility Fluctuations in Semiconductors. SPIE 4th International Symposium Fluctuation and Noise, Florence, 20-24 May 2007, Vol. 6600, 66001K-(1-8).

[22] Gasparyan, F.V., Vitusevich, S.A., Offenhäusser, A. and Schöning, M.J. (2011) Modified Charge Fluctuation Noise Model for Electrolyte-Insulator-Semiconductor Devices. Modern Physics Letters B, 25, 831-840. https://doi.org/10.1142/S0217984911026103

[23] Gasparyan, F.V. and Aroutiounian, V.M. (2015) Quantum Modulation of the Channel Charge and Distributed Capacitance of Double Gated Nanosize FETs. Advances in Nano Research, 3, 49-54. https://doi.org/10.12989/anr.2015.3.1.049

[24] Hassibi, A., Navid, R., Dutton, R.W. and Lee, T.H. (2004) Comprehensive Study of Noise Processes in Electrode Electrolyte Interfaces. Journal of Applied Physics, 96, 1074-1082. https://doi.org/10.1063/1.1755429

[25] Tyagai, V.A. (1971) Faradaic Noise of Complex Electrochemical Reactions. Electrochimica Acta, 16, 1647-1654. https://doi.org/10.1016/0013-4686(71)85075-2

[26] Deen, M.J., Shinwari, M.W. and Ranuarez, J.C. (2006) Noise Considerations in Field-Effect Biosensors. Journal of Applied Physics, 100, Article ID: 074703(1-8). https://doi.org/10.1063/1.2355542

[27] Massobrio, G., Martinoa, S. and Grattarola, M. (1992) Light-Addressable Chemical Sensors: Modeling and Computer Simulations. Sensors and Actuators B, 7, 484-487. https://doi.org/10.1038/nchembio.611

[28] Gasparyan, F.V. (2010) Excess Noises in (Bio-)chemical Nanoscale Sensors. Sensors \& Transducers Journal, 122, 72-84.

[29] Hassibi, A., Zahedi, S., Navid, R., Dutton, R.W. and Lee, T.H. (2005) Biological Shot-Noise and Quantum-Limited Signal-to-Noise Ratio in Affinity-Based Biosensors. Journal of Applied Physics, 97, Article ID: 084701(1-9). https://doi.org/10.1063/1.1861970

[30] Hooge, F.N. (1969) 1/f Noise Is No Surface Effect. Physics Letters A, 29, 139-140. https://doi.org/10.1016/0375-9601(69)90076-0

[31] Hooge, F.N., Kleinpenning, T.G.M. and Vandamme, L.K.J. (1981) Experimental Studies on 1/f Noise. Reports on Progress in Physics, 44, 479-532. https://iopscience.iop.org/article/10.1088/0034-4885/44/5/001/pdf https://doi.org/10.1088/0034-4885/44/5/001

[32] Hung, K.K., Ko, P.K., Hu, C. and Cheng, Y.C. (1990) A Unified Model for the Flicker Noise in Metal-Oxide-Semiconductor Field-Effect Transistors. IEEE Transactions on Electron Devices, 37, 654-665. https://doi.org/10.1109/16.47770

[33] Christensson, S., Lundström, I. and Svensson, C. (1968) Low Frequency Noise in 
MOS Transistors I. Theory. Solid-State Electronics, 11, 797-812. https://doi.org/10.1016/0038-1101(68)90100-7

[34] Christensson, S. and Lundström, I. (1968) Low Frequency Noise in MOS Transistors II. Experiments. Solid-State Electronics, 11, 813-820.

https://doi.org/10.1016/0038-1101(68)90101-9

[35] Gasparyan, L., Mazo, I., Simonyan, V. and Gasparyan, F. (2019) DNA Sequencing: Current State and Prospects of Development. Open Journal of Biophysics, 9, 169-197. https://doi.org/10.4236/ojbiphy.2019.93013

[36] Gasparyan, L., Mazo, I., Simonyan, V. and Gasparyan, F. (2020) EIS Biosensor for Detection of DNA Low Concentration. Journal of Contemporary Physics (Armenian Academy of Sciences). (In Press)

[37] Pantelides, S.T., Wang, S., Franceschetti, A., et al. (2006) $\mathrm{Si} / \mathrm{SiO}_{2}$ and $\mathrm{SiC} / \mathrm{SiO}_{2} \mathrm{In}$ terfaces for MOSFETs-Challenges and Advances. Materials Science Forum, 527-529, 935-948.

[38] Jeong, C., Antoniadis, D. and Lundstrom, M.S. (2009) On Backscattering and Mobility in Nanoscale Silicon MOSFETs. IEEE Transactions on Electron Devices, 56, 2762-2769. https://doi.org/10.1109/TED.2009.2030844

[39] Park, C., Lee, C., Lee, K., Moon, B.-J., Byun, Y.H. and Shur, M. (1991) A Unified Current-Voltage Model for Long-Channel nMOSFETs. IEEE Transactions on Electron Devices, 38, 399-406. https://doi.org/10.1109/16.69923

[40] Gildenblat, G.S. and Huang, C.-L. (1989) Engineering Model of Inversion Channel Mobility for 60-300k Temperature Range. Electronics Letters, 25, 634-636. https://doi.org/10.1049/el:19890430

[41] Pud, S., Gasparyan, F., Petrychuk, M., Li, J., Offenhausser, A. and Vitusevich, S.A. (2014) Single Trap Dynamics in Electrolyte-Gated Si-Nanowire Field Effect Transistors. Journal of Applied Physics, 115, Article ID: 233705(1-11). https://doi.org/10.1063/1.4960704 